\title{
Boas práticas na assistência à saúde: bundle para prevenção de pneumonia associada à ventilação mecânica
}

\author{
Good practices in health care: bundle for prevention of ventilator-associated pneumonia \\ Buenas prácticas en la asistencia a la salud: bundle para prevención de neumonía \\ asociada a la ventilación
}

Camilla Cristina Lisboa do Nascimento ${ }^{1 *}$, Regiane Camarão Farias ${ }^{1}$, Marcelo Williams Oliveira de Souza ${ }^{2}$.

\section{RESUMO}

Objetivo: Construir um bundle para prevenção de pneumonia associada à ventilação mecânica (PAVM) em Unidade de Terapia Intensiva (UTI). Métodos: Trata-se de um estudo metodológico, para criação de um bundle de PAVM para profissionais da saúde de uma UTI de um Hospital de Referência, localizado em BelémPA. A tecnologia foi construída por meio de levantamento bibliográfico que teve como finalidade agrupar informações sobre assistência aos pacientes com PAVM. A partir disso, foram destacadas as principais medidas para a construção do bundle. Resultados: Foi construindo um bundle sobre PAVM com as principais medidas de prevenção: higiene das mãos do profissional, decúbito elevado em $30^{\circ}$ a $45^{\circ}$ graus, adequação do nível de sedação, aspiração endotraqueal, higienização oral, mudança de circuito, monitoração da pressão de cuff e evitar a reitubação. Conclusão: É necessário que a equipe multiprofissional tenha o conhecimento teórico-prático das ações para prevenir e minimizar a incidência desses acometimentos. Espera-se que o estudo colabore com as boas práticas na assistência em saúde.

Palavras-chave: Infecção Hospitalar, Assistência Hospitalar, Unidades de Terapia Intensiva.

\begin{abstract}
Objective: To construct a bundle for the prevention of ventilator-associated pneumonia (VMR) in the intensive Care unit (ICU). Methods: This is a methodological study for the creation of a bundle of VMR for health professionals from an ICU of a referral Hospital, located in Belém-PA. The technology was built by means of a bibliographic survey that had the purpose of grouping information on assistance to patients with VME. From this, the main measures for the construction of the bundle were highlighted. Results: It was constructing a bundle on VMR with the main preventive measures: hygiene of the professional's hands, elevated decubitus at $30 \circ$ to 45 degrees, adequacy of the level of sedation, endotracheal aspiration, oral hygiene, change of circuit, monitoring of cuff pressure and avoiding reintubation. Conclusion: It is necessary that the multidisciplinary team have the theoretical and practical knowledge of the actions to prevent and minimize the incidence of these affections. The study is expected to collaborate with good practices in health care.
\end{abstract}

Key words: Cross Infection, Hospital Care, Intensive Care Units.

\section{RESUMEN}

Objetivo: construir un bundle para la prevención de la neumonía asociada al ventilador (VMR) en la unidad de cuidados intensivos ( $\mathrm{UCl}$ ). Métodos: se trata de un estudio metodológico para la creación de un bundle de VMR para profesionales sanitarios de una $\mathrm{UCl}$ de un hospital de referencia, ubicado en Belém-PA. La

${ }^{1}$ Universidade do Estado do Pará (UEPA), Belém-PA. *E-mail: camilla.nasc@gmail.com

2 Universidade Federal do Pará, Belém-PA.

SUBMETIDO EM: 2/2019

ACEITO EM: 3/2019

PUBLICADO EM: 5/2019

REAS/EJCH | Vol. Sup. 23 | e431| DOI: https://doi.org/10.25248/reas.e431.2019 Página 1 de 6 
tecnología fue construida por medio de un estudio bibliográfico que tenía el propósito de agrupar información sobre la asistencia a pacientes con VME. De ahí se destacaron las principales medidas para la construcción del bundle. Resultados: se estaba construyendo un paquete en VMR con las principales medidas preventivas: higiene de las manos del profesional, elevada decúbito a $30^{\circ}$ a 45 grados, adecuación del nivel de sedación, aspiración endotraqueal, higiene bucal, cambio de circuito, monitorización de la presión del cuff y evitar la reitubation. Conclusión: es necesario que el equipo multidisciplinar tenga el conocimiento teórico y práctico de las acciones para prevenir y minimizar la incidencia de estos afectos. Se espera que el estudio colabore con buenas prácticas en el cuidado de la salud.

Palabras clave: Infección Hospitalaria, Atención Hospitalaria, Unidades de Cuidados Intensivos.

\section{INTRODUÇÃO}

As Infecções Relacionadas à Assistência à Saúde (IRAS) são consideradas como um problema de saúde pública por serem conceituadas como eventos adversos adquiridos durante a prestação dos cuidados em saúde e, por isso, se apresentam como riscos significativos à segurança do paciente (BRASIL, 2017).

As IRAS apresentam impacto sobre letalidade hospitalar, duração da internação e custos. O aumento das condições que induzem à internação de indivíduos cada vez mais grave e imunocomprometidos confere às IRAS especial relevância para a saúde pública. Além disso, os países em desenvolvimento sofrem com maior carga de IRAS, podendo ser até 20 vezes superior aos países desenvolvidos (PADOVEZE MC, FORTALEZA CMCB, 2014).

Ressalta-se que as IRAS atingem em média 14\% das internações em nível nacional e dos 234 milhões de pacientes que são operados por ano no mundo, em média, um milhão morre em decorrência destas (SILVA AN, et al., 2016).

O alto risco de mortalidade relacionada às IRAS está intimamente associado a fatores como a realização de procedimentos invasivos diagnósticos e terapêuticos, à gravidade da doença de base que acomete o paciente, ao sítio da infecção, à adequação da terapia e à sensibilidade dos microrganismos aos antimicrobianos (SOUZA ES, et al., 2015).

O trabalho em Unidades de Terapia Intensiva (UTI) é peculiar, pois as condições clínicas dos pacientes são oscilantes, exigindo atenção ainda maior da equipe multiprofissional (PEDREIRA LC, et al., 2013). A prevalência destas infecções varia com o tipo de UTI e, portanto, com a natureza dos pacientes. Em UTIs gerais, as infecções urinárias, respiratórias e associadas a cateteres vasculares são as mais frequentes (BRASIL, 2017).

Pacientes que necessitam de Ventilação Mecânica (VM) são frequentes nas UTI e, de acordo com Azevedo LCP et al. (2015), a Pneumonia associada à Ventilação Mecânica (PAV) é a segunda infecção nosocomial mais frequente na população crítica e a mais frequente entre os pacientes ventilados. Além de doença, a PAV é cada vez mais considerada um marcador da qualidade de assistência à saúde por ser uma doença sujeita a prevenção.

Nesse sentido, foram criadas em 2017 as Medidas de Prevenção de Infecção Relacionada à Assistência à Saúde pela Agência Nacional de Vigilância Sanitária, com o intuito de reduzir a incidência das IRAS, a partir da disponibilização das principais medidas preventivas práticas adequadas à realidade brasileira.

Destaca-se então a criação de Tecnologias Educacionais (TE) em saúde com um olhar direcionado para a equipe multiprofissional, uma vez que, por meio dessa, é possível visualizar melhor informações, potencializando o conhecimento desta, atuando no processo de ensino-aprendizagem na educação permanente do profissional de saúde (SILVA LHF, SANTO FHE, 2013).

As tecnologias podem ser classificadas em Tecnologias Educacionais (TE): dispositivos para a mediação de processos de ensinar e aprender; Tecnologias Assistenciais: dispositivos para a mediação de processos

REAS/EJCH | Vol. Sup. 23 | e431 | DOI: https://doi.org/10.25248/reas.e431.2019 Página 2 de 6 
de cuidar; e Tecnologias Gerenciais: dispositivos para mediação de processos de gestão nos diversos sistemas de saúde (MANIVA SJCF, et al., 2018). O uso de TE, entendidas como ferramentas facilitadoras na promoção de um cuidado humanizado, potencializa a educação e a orientação dos cuidados (CARDOSO RSS, et al., 2018).

Com isso, destaca-se a construção de pacote de intervenções, denominado bundles, que se apresenta como um conjunto de medidas baseadas em evidências científicas integradas para prática de prevenções dentro da assistência em saúde (SILVA AG e OLIVEIRA AC, 2018).

Tendo em vista a complexidade e a gravidade das IRAS, demonstradas pelos altos índices de mortalidade, a necessidade de prevenção como estratégia de promoção da segurança do paciente e a contribuição direta da enfermagem no controle dessas infecções (SOUZA ES, et al., 2015), esse trabalho tem como objetivo construir uma tecnologia educacional, por meio de um bundle, sobre medidas preventivas para o controle das IRAS na UTI.

\section{MÉTODOS}

O trabalho trata-se de um estudo metodológico. Esse estudo estabelece às etapas de estabelecimento da estrutura conceitual; definição dos objetivos e população; construção dos itens; seleção e organização dos itens; e estruturação do instrumento (LEITE SS, 2018).

A tecnologia educacional foi direcionada para uma Unidade de Terapia Intensiva de um hospital, localizado na cidade de Belém - PA. O hospital é atualmente referência estadual em Psiquiatria, Cardiologia e Nefrologia e tem como missão de garantir atendimento ambulatorial e hospitalar de média e alta complexidade, além de apoiar o ensino e pesquisa na área de saúde. A Unidade de Terapia Intensiva apresenta 12 leitos, contando com 6 equipes multiprofissionais que é composta por 11 enfermeiros, 26 técnicos de enfermagem, 11 médicos, 4 fisioterapeutas, 1 nutricionista, 1 psicólogo, além de 2 postos de enfermagem, 1 quarto de isolamento e 1 sala de higienização.

A TE foi construída por meio de levantamento bibliográfico que teve como finalidade agrupar informações sobre assistência aos pacientes com PAV. Foram levados em consideração artigos publicados em português, entre os anos de 2013 a 2018, completos e gratuitos. Além disso, também foram utilizados livros clássicos da bibliográfica estudada.

Foram utilizadas as bases de dados: Literatura Latino-Americana e do Caribe em Ciências da Saúde (LILACS), Bases de Dados de Enfermagem (BDENF) e os descritores: "Pneumonia Associado à Ventilação Mecânica AND Prevenção de Doenças AND Unidades de Terapia Intensiva". A partir do levantamento de dados, foram identificadas, reunidas e sintetizadas as melhores evidências disponíveis sobre o tema de acordo com os critérios de inclusão, e suas formas de prevenção atreladas à assistência à saúde para a construção da tecnologia a ser implantada na UTI. Toda a coleta foi agrupada e organizada no programa Excel 2007.

Após construção dessas TEs em saúde, foram agendadas apresentações à equipe multiprofissional, realizadas na própria Unidade de Terapia Intensiva, explicando os objetivos do estudo e a importância da utilização das TEs durante os procedimentos diários dentro da assistência à saúde. O convite à participação da equipe foi realizado em intervalos de trabalho na Unidade de Terapia Intensiva, levando considerando os momentos mais propícios para apresentação. Ressalta-se que por se tratar de construção de TE com base na literatura, não houve necessidade de aprovação do Comitê de Ética. No entanto, o projeto de construção do material obteve autorização institucional para a sua realização.

\section{RESULTADOS E DISCUSSÃO}

Foram encontrados 55 artigos, após aplicar o critério de exclusão, foram utilizados 23 artigos que tratavam sobre a temática. A partir do levantamento bibliográfico, foi possível identificar quais as principais medidas de prevenção de PAV. As medidas adotadas na TE, por meio de bundle, apresentam-se na figura 1. 
Figura 1 - Bundle de prevenção de PAVM. Belém-PA, 2018.

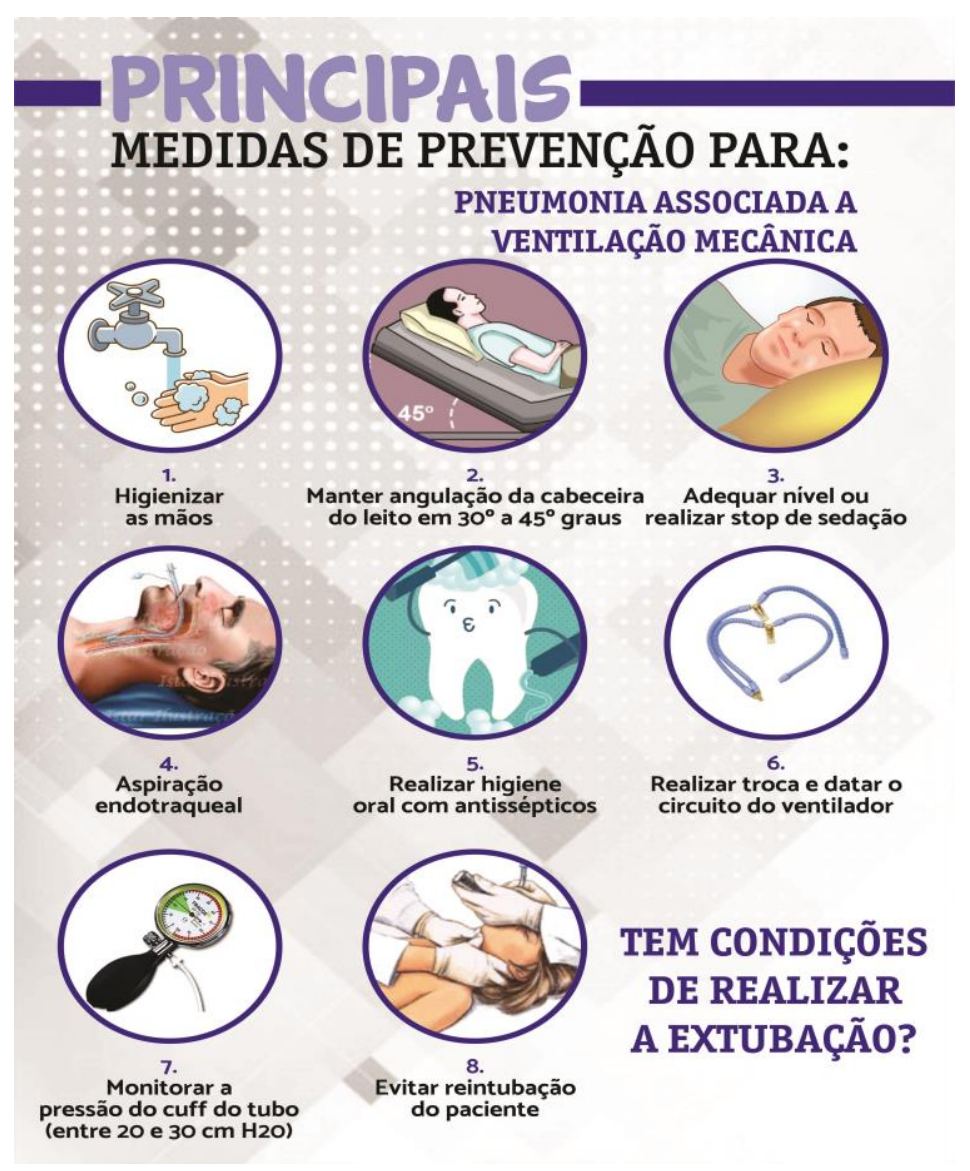

Fonte: Dados da pesquisa, 2018

A higienização das mãos destacada como medida primordial para o controle de infecções relacionadas à assistência à saúde. Por este motivo, é vista como forma para o controle de IRAS, incluindo aquelas decorrentes da transmissão cruzada de microrganismos multirresistentes (BRASIL, 2017).

Almeida WB et al. (2018) enfatiza em seus estudos sobre a importância da lavagem das mãos dentro da UTI, por ser tratar de um local que realizam procedimentos invasivos em pacientes em estado grave, patologias de base primárias, secundárias ou terciárias. A conduta de realizar a antissepsia das mãos antes e após o manuseio de pacientes é de extrema importância para prevenir a propagação de IRAS.

Quanto à modificação na angulação da cabeceira do leito para 30 a 45ํㅡㅁaus, estudos apontam quando o decúbito do paciente não é trocado durante uma periodicidade, o processo inflamatório junto com o peso do pulmão favorece a criação de zonas dependentes, com queda da complacência e aumento da resistência e essa redução da complacência está associada com aumento do risco de morte (SILVA SG, et al., 2012). Isto é, a posição do paciente afeta diretamente a mecânica do sistema respiratório quando este está submetido à VM. Por isso, é necessário preconizar posições que favoreçam a redução cargas mecânicas opositoras a entrada de ar para dá maior estabilidade do ar nos alvéolos.

Já sobre a adequação da sedação, ela deve ser diminuída ou pausada sempre que possível. Maniva SJCF et al. (2018) aponta que com a interrupção ou diminuição da sedação, é possível realizar uma avaliação adequada do padrão respiratório do paciente, verificando se ele pode ser extubado, reduzindo o tempo de ventilação mecânica e consequentemente a incidência de PAV.

A aspiração endotraqueal é de grande importância para a diminuição do acúmulo de secreção na subglótica. Quando o paciente é exposto a VM, acaba apresentando um aumento de fatores de riscos, pois o 
paciente perde da barreira natural entre orofaringe e a traqueia e, se sedados, ficam desprovidos do reflexo da tosse, acumulando secreções acima do cuff da cânula endotraqueal (SILVA SG et al., 2012).

Esse cuidado deve ser feito rotineiramente e quando necessário, além de ser realizada com critérios e guiada por cuidados específicos para que não traga prejuízos ao paciente.

A utilização de clorexidina na higiene oral de pacientes em VM diminui a colonização da cavidade bucal, podendo reduzir a incidência da PAV. Além disso, estudos apontam que esse procedimento é seguro, de baixo custo e tolerável (BERALDO CC e ANDRADE D, 2008).

Já sobre a realização da troca do circuito do ventilador, segundo Brasil (2017) a troca deste não deve ser menor de 48 horas para diminuir o risco de contaminação com o ambiente. No entanto, o descarte do líquido condensado e a desinfecção do alto do circuito deve ser realizada periodicamente. Vale ressaltar que todos os procedimentos realizados devem ser datados e evoluídos em prontuário para garantir a segurança do paciente.

Brasil (2009) recomenda que a pressão do cuff do tubo endotraqueal deve ser mantida entre 20 a $30 \mathrm{~cm}$ $\mathrm{H}_{2} \mathrm{O}$. Com essa pressão, é possível que prevenir possíveis lesões endotraqueais, além de evitar que a secreção da região subglótica escorra ao redor do balonete alcançando o pulmão (MARAN E et al., 2019).

Quanto evitar a reintubação do paciente, Kalil AC et al. (2016) destaca em seus estudos que reexpor o paciente a VM aumenta o risco para o surgimento de PAV. Isto é, quando o paciente não tolera o teste de respiração espontânea é necessário que seja realizado um planejamento a estratégia a ser adotada a seguir - nova tentativa de interrupção da ventilação mecânica ou desmame gradual.

Além desses tópicos, o bundle enfatiza a pergunta "Tem condições de realizar a extubação?". Esse questionamento vem como forma de salientar a equipe multiprofissional ter o olhar de retirar a VM dos pacientes, quando for possível. A literatura mostra que o tempo prolongado maior de sete dias com VM, já é indicativo de paciente em fator de risco para IRAS (POMBO CMN et al., 2010).

Com a construção finalizada, houve a exposição do produto e o objetivo do estudo para a equipe multiprofissional atuante no setor onde ocorreu o estudo, apresentando dados significativos sobre índices de infecção causados por falhas nas condutas de boas práticas na assistência à saúde. Houve também esclarecimentos de dúvidas quanto as IRAS e as práticas de prevenção abordadas pela TE em saúde. Por fim, a TE foi anexada em pontos estratégicos, de fácil acesso e visualização no setor, para a equipe multiprofissional.

\section{CONSIDERAÇÕES FINAIS}

A equipe de enfermagem é a maior responsável pela assistência prestada aos pacientes em UTI, sendo estes quem mais conhecem as necessidades do paciente e estão em contato direto e contínuo, fazendo-se imprescindível o conhecimento teórico-prático dessas ações para prevenir e minimizar a incidência desses acometimentos. Espera-se que esta pesquisa possa contribuir para a prevenção e controle de infecções relacionadas à assistência à saúde na Unidade de Terapia Intensiva e a realização de mais estudos voltados para essa temática.

\section{REFERÊNCIAS}

1. ALMEIDA WB, et al. Infecção hospitalar: controle e disseminação nas mãos dos profissionais de saúde de uma Unidade de Terapia Intensiva. Revista Eletrônica Acervo Saúde, 2018; 11(2): e130.

2. AZEVEDO LCP, et al. Medicina intensiva: abordagem prática. 2nd ed. Barueri: Manole, 2015; 1184p.

3. BARDIN L. Análise de Conteúdo. 1nd ed. São Paulo: Edições 70 - Brasil, 2011; 280p.

4. BERALDO CC, ANDRADE D. Higiene bucal com clorexidina na prevenção de pneumonia associada à ventilação mecânica. Jornal Brasileiro de Pneumonia, 2008; 34(9): 707-714.

5. BRASIL. Agência Nacional de Vigilância Sanitária. Medidas de Prevenção de Infecção Relacionada à Assistência à Saúde. 2nd ed. Brasília: Anvisa, 2017; 122p. 
6. BRASIL. Agência Nacional de Vigilância Sanitária. Infecções do trato respiratório: orientações para prevenção de infecções relacionadas à assistência à saúde. 1nd ed. Brasília: Anvisa, 2009; 27p.

7. CARDOSO RSS, et al. Tecnologia educacional: um instrumento dinamizador do cuidado com idosos. Revista Brasileira de Enfermagem, 2018; 71(2): 839-845.

8. KALIL AC, et al. Management of Adults With Hospital-acquired and Ventilator-associated Pneumonia: 2016 Clinical Practice Guidelines by the Infectious Diseases Society of America and the American Thoracic Society. Clin Infect Dis, 2016; 63(5): 61-111.

9. LEITE SS, et al. Construção e validação de Instrumento de Validação de Conteúdo Educativo em Saúde. Revista Brasileira de Enfermagem, 2018; 71(4): 1635-1641.

10. MANIVA SJCF, et al. Tecnologias educativas para educação em saúde no acidente vascular cerebral: revisão integrativa. Revista Brasileira de Enfermagem, 2018; 71 (4): 1724-1731.

11. PADOVEZE MC, FORTALEZA CMCB. Infecções relacionadas à assistência à saúde: desafios para a saúde pública no Brasil. Revista Saúde Pública, 2014; 48(6): 995-1001.

12. PEDREIRA LC, et al. Evento adverso no idoso em Unidade de Terapia Intensiva. Revista Brasileira de Enfermagem. Brasília, 2013; 66(3): 429-436.

13. $\mathrm{POMBO}, \mathrm{CMN}$, et al. Conhecimento dos profissionais de saúde na Unidade de Terapia Intensiva sobre prevenção de pneumonia associada à ventilação mecânica. Ciência \& Saúde Coletiva, 2010: 15(1): 1061-1072.

14. RAMOS NSO, ALVES GR. A importância dos cuidados da equipe de enfermagem na prevenção da pneumonia associada a ventilação mecânica (PAVM): revisão da literatura. Revista Científica FacMais, 2018; 3(1): 30-38.

15. SILVA AG, OLIVEIRA AC. Impacto da implementação dos bundles na redução das infecções da corrente sanguínea: Uma revisão integrativa. Texto Contexto Enfermagem, 2018; 27(1): 1-13.

16. SILVA AN, et al. Medidas de prevenção à infecção hospitalar: uma revisão livre na literatura. Monografia de Especialização (Pós-Graduação em Fisioterapia Hospitalar) - Escola Bahiana de Medicina e Saúde Pública, Bahia, 2016; 12p.

17. SILVA LHF, SANTO FHE. Tecnologia educacional interativa como estratégia de educação permanente: um estudo descritivo. Online Brazilian Journal of Nursing, 2013; 12(1): 701-703.

18. SILVA SG, et al. Bundle de prevenção da pneumonia associada à ventilação mecânica: uma construção coletiva. Texto Contexto Enfermagem, 2012; 21(4): 837-844.

19. SOUZA ES, et al. Mortalidade e riscos associados a infecção relacionada à assistência à saúde. Texto Contexto Enfermagem, 2015; 24(1): 220-228. 